\title{
Contingency Management Intervention in Quitting Substance Abuse: A Literature Review
}

\author{
Tianrun Zhu
}

\author{
University of British Columbia, Faculty of Science, Vancouver, V6T 1 Z4 \\ *Corresponding author. Email: tzhu05@student.ubc.ca
}

\begin{abstract}
Contingency management (CM) intervention is a commonly used and highly effective method in treating substance abuse. In CM intervention, customers are positively reinforced if they follow the combined treatment and make progress in quitting substance abuse. The type of positive reinforcers is usually monetary based. Sometimes other types of positive reinforcers are also used. Since the reinforcers are monetary-based, it is important to analyze the cost-effectiveness of these various reinforcers. Previous researches have developed many kinds of reinforcers and new types of reinforcers are under development. Researchers have combined CM intervention with many other treatment methods to increase the effectiveness of the treatment. In this literature review, the author analyzed previous studies on CM interventions, including CM and smoking, CM and binge drinking, and CM and drug abuse. In conclusion, $\mathrm{CM}$ intervention is effective and recommended in treating substance abuse, and is useful to various groups of people. Some problems that occurred when using CM intervention need further researches to answer. For instance, the efficacy of CM intervention can be decreased if the intervention is not directly addressed to the drinking behaviour.
\end{abstract}

Keywords : contingency management, positive reinforcers, smoking cessation, alcohol abstinence, drug abstinence

\section{INTRODUCTION}

Substance abuse has been a serious issue in modern society. Various methods have been developed to help quit substance abuse, including the contingency management (CM) intervention which consists of the utilization of positive reinforcers. By giving positive reinforcers when the customers behave as the treatment requires, the customers tend to be more motivated to follow the treatment in the future, thus having a higher potential to quit substance abuse. Positive reinforcers are successful in reducing the use of a variety of substances, including tobacco, alcohol, marijuana, cocaine, and opiates. There is evidence demonstrating that treatments consisting of positive reinforcers are more effective than treatments that do not use positive reinforcers[1].

Most positive reinforcers used are monetarybased[1], sometimes other types of positive reinforcers, such as television viewing, are also developed in response to certain cases. Even monetary-based reinforcers are not all the same. For instance, the reinforcers can be money or items which the customers like. Also, it is of great importance to find the positive reinforcers that cost less while still be effective in a specific treatment. It is important to know if all groups of people are suitable for CM intervention. For example, if $\mathrm{CM}$ intervention is effective when used to patients with mental illness. Since different positive reinforcers are used in different cases, it is instructive to analysis the various positive reinforcers to make better use of them in the future. This article will be a literature review which will analysis the use of $\mathrm{CM}$ intervention from previous researches.

This article aims to analyzing the effectiveness of the variety of positive reinforcers used in $\mathrm{CM}$ for smoking cessation, alcohol abstinence and drug abstinence, including the process of usage, the cost-effectiveness of the reinforcers and the behaviour they should be addressed in order to gain a better therapeutic effect.

\section{CONTINGENCY MANAGEMENT IN QUITTING SMOKING}

Growing evidence has suggested that various kinds of positive reinforcers can be useful in quitting smoking. In a pilot study conducted by Dunn et al [2], methadone- 
maintained participants were recruited to take part in a smoking cessation program. In this research, the participants were taking both methadone maintenance and CM intervention for smoking cessation. The research aimed to determine whether contingency management intervention can promote quitting smoking among methadone-maintained participants. The participants were divided into contingent or noncontingent groups, and vouchers were given to participants in the contingent group if they showed biological evidence of smoking cessation while participants in the noncontingent group received vouchers independent of their progress on smoking cessation. The study found that voucher-based CM intervention can encourage methadone-maintained patients to stick on the treatment of smoking cessation.

Stoops et al [3] conducted research that demonstrated that $\mathrm{CM}$ intervention can help smoking cessation in combination with an Internet-based monitoring program. The positive reinforcer used in this contingency management intervention was monetary-based, and the Internet-based monitoring program was designed to track the participants living in the rural area for whom it can be difficult to be monitored through in-person methods. The participants were asked to upload videos of them taking the $\mathrm{CO}$ test and post the test results. The participants were randomly categorized into the Abstinence Contingent (AC) group in which they received monetary incentives according to their recent contingency on smoking abstinence and the Yoked Control (YC) group in which the incentives were given independently of their smoking status. There are two major findings in this research. One is that participants in the $\mathrm{AC}$ group were more likely to have negative $\mathrm{CO}$ results and post the sample on the website than participants in the YC groups. The other is that participants in the AC group also had a higher potential to maintain certain level of smoking abstinence during the 6-week intervention than participants in the YC group. The results illustrated that it is feasible to increase short-term smoking abstinence in rural populations through giving positive reinforcement over Internet. Another study that combined remote monitoring and CM intervention used a smartphone app as the CO monitor and delivered 10 US dollars to the participants every day they achieved smoking abstinence [4]. This study obtained the conclusion that positive reinforcers can help maintain smoking abstinence, and the researchers also suggested that money as a positive reinforcer is especially effective to people who live in rural area or have low incomes.

To sum up, positive reinforcers used in smoking cessation are mainly monetary-based, and they increase the effectiveness of the programs that are combined with them. The CM intervention can be combined with both in-person or online programs. The monetary-based positive reinforcers are especially useful when the participant are from rural area where people have less level of income. Monetary-based reinforcers are effective when the participants are from low income communities. Another point to conclude is that relatively speaking, the m.onetary-based reinforcers used in the researches mentioned above are not costly.

\section{CONTINGENCY MANAGEMENT IN QUITTING BINGE DRINKING}

There are also attempts to use positive reinforcers in quitting binge drinking. Among all age groups, young adultsage from 18 to 24 years consume the highest amount of alcohol. Generally speaking, the less activity they engage in without alcohol, the more alcohol they consume. One study used exercise to make intervene with college students who had engaged in binge drinking as a result of their sedentary living style [5]. The participants were instructed to motivational enhancement therapy (MET) which is an effective method to increase exercise. The participants were divided into two groups, one with MET alone and the other with MET and CM intervention. The positive reinforcers used in this study were tangible items. The results of this study illustrated that compared with the participants in the MET group, the participants in the MET+CM group self-reported a higher frequency of exercise. However, participants in the two groups showed no difference in other aspects of exercise, physical fitness and alcohol consumption before and after the intervention. One of the possible reasons is that the $\mathrm{CM}$ intervention was not addressed the drinking behaviour directly. Further investigation should be made to figure out this phenomenon.

One study aimed at evaluating the efficacy of $\mathrm{CM}$ intervention in reducing alcohol consumption reached the conclusion that $\mathrm{CM}$ is effective [6]. Participants in this study all have the problem of heavy drinking. The trial went for 3 weeks, and the participants were asked to wear a Secure Continuous Remote Alcohol Monitoring (SCRAM) bracelet and report the use of alcohol and drugs. In the first week, the participants were asked to consume as much alcohol as they usually do. In the second and third week, they were reinforced not to drink alcohol by money. They would get the money if they did not drink any alcohol on that day. As days passed, the money given increased from $\$ 5$ to $\$ 17$. Both selfreported and SCRAM detected alcohol consumption decreased to a large degree in the second and third week, which demonstrates that $\mathrm{CM}$ intervention is effective in reducing alcohol consumption. Compared to the study above, this study shows that CM intervention influences alcohol use when it was performed directly to drinking problem instead of exercising.

McDonell et al [7] research with the purpose to determine if $\mathrm{CM}$ intervention promotes alcohol abstinence of outpatients with serious co-occurring mental illnesses. The participants went through 4 weeks 
of observation then were randomly assigned to a 12week $\mathrm{CM}$ intervention. Alcohol consumption was measured by ethyl glucuronide (EtG) alcohol biomarker. CM intervention consisted of three" prize draw" processes a week when the outpatient had EtG-negative samples and gift cards given to the outpatients if they attend the treatment. Compared to the control group, outpatients who received CM intervention had much lower mean EtG levels, fewer self-reported drinking and binge drinking experiences and more EtG-negative samples submitted. The study showed that CM intervention can help patients with serious mental illnesses to reduce the their drinking behaviour.

To sum up, CM intervention can be useful in quitting binge drinking. However, its efficacy may be influenced if it is not directly addressed to the drinking behaviour. $\mathrm{CM}$ intervention is also useful for patients with mental illnesses. These previous researches demonstrate that, in quitting binge drinking, $\mathrm{CM}$ intervention can be used in a wide range of situations and remain to be effective. However, it should be noticed that CM intervention may not be helpful if wrong behaviour is positively reinforced.

\section{CONTINGENCY MANAGEMENT IN QUITTING DRUG ABUSE}

Researches have developed several treatments that combine CM intervention with other methods to help drug abusers to quit. In one study conducted by Litt et al [8], the researchers combined MET, cognitivebehavioral coping skills training (CBT) and CM in order to build a treatment which is able to increase the participant's self-efficacy when quitting marijuana. In this research, they assigned the participants into four conditions: a case management control condition, MET/CBT, CM alone and MET/CBT + CM. $\mathrm{MET} / \mathrm{CBT}+\mathrm{CM}$ was the most effective condition in quitting marijuana, even in long term. MET/CBT was also very effective but was less than MET/CBT + $\mathrm{CM}$. The researchers suggested MET/CBT $+\mathrm{CM}$ and MET/CBT conditions were the most effective ones because they increase the self-efficacy of the participants. Although it is not known whether CM intervention can increase self-efficacy, it is clear that CM intervention can act as a promotor in a treatment.

Olmstead and Petry [9] conducted research aimed at investigating the cost-effectiveness of prize-based and voucher-based CM used in quitting cocaine and heroin. The CM interventions evaluated in this research were those added to other standard treatments (ST). The participants in this study were outpatients from community treatment centers. The participants were randomly divided into two groups receiving ST with prizes (prize CM) and ST with vouchers (voucher CM). The treatments went for 12 weeks. The effectiveness of the treatment was measured by the primary patient outcome was the longest duration of confirmed abstinence (LDA) from the substance. The unit costs of the prizes and the vouchers were also collected. Then the researchers calculated the incremental cost-effectiveness ratios and the acceptability curves, according to which Prize CM was more cost-effective than voucher CM. Since most CM interventions use monetary-based positive reinforcers, it is instructive to investigate the cost-effectiveness of different positive reinforcers.

Metrebian et al [10]. conducted research in the UK and suggested that $\mathrm{CM}$ intervention should be included in the drug treatments in the UK. The objects in this experiment were those who used heroin and other opiates. Through investigation, they suggested that it would bring great benefit if $\mathrm{CM}$ intervention was popularized in treating the abuse of heroin and other opiate. It is important that there is potential that $\mathrm{CM}$ interventions can be popularized to a size of a country.

To sum up, in treating drug abuse, CM intervention is effective and worth popularizing. Some positive reinforcers are more cost-effective than others. Since most positive reinforcers used in $\mathrm{CM}$ intervention are monetary-based, it is important to choose the most costeffective reinforcer in treatment. The increase of costeffectiveness may correspondingly help the popularization of $\mathrm{CM}$ intervention. $\mathrm{CM}$ intervention can be combined with other types of treatment when treating drug abuse, especially those treatments that can increase the participants' self-efficacy.

\section{CONCLUSION}

From previous researches, it is clear that $\mathrm{CM}$ interventions are highly effective and recommended to use treatments of tobacco, alcohol and drug abuse. CM interventions are usually additions to other kinds of treatments, such as MET. However, it sometimes can also be used alone with still high efficacy. CM intervention can be used for various groups of people. Mental illnesses do not influence how people are affected by $\mathrm{CM}$ interventions. Most positive reinforcers used in CM intervention are monetary-based, so it is of great significance to understand the cost-effectiveness of different positive reinforcers used. For certain cases, some reinforcers can be more effective than others. Sometimes the reinforcers are individualized, which means that the individuals has choices to choose the reinforcer among a pool of reinforcers. $\mathrm{CM}$ intervention may not be effective if it is not addressed to the abusing behaviour directly. CM interventions have been confirmed to have strong short-term efficacy, while the long-term efficacy still needs to the further analyzed.

Future research on CM intervention may focus on its long-term efficacy. For long-term efficacy, more research is needed to determine whether $\mathrm{CM}$ needs to directly target target behaviors. In the future, researchers 
may develop more types of positive enhancers for CM intervention, and the cost-effectiveness of different enhancers can also be compared. In fact, In fact, the more cost-effective a CM intervention is, the easier it is to promote it.

\section{ACKNOWLEDGMENT}

I am grateful to my family and my teachers who supported me when doing this research. I also appreciated UBC Library from which I got access to a wide range of papers.

\section{REFERENCES}

[1] HIGGINS, S. T., HEIL, S. H., \& LUSSIER, J. P. (2004). Clinical implications of reinforcement as a determinant of substance use disorders. Annual Review of Psychology, 55(1), 431-461. https://doi.org/ $10.1146 /$ annurev.psych.55.090902.142033

[2] Dunn, K. E., Sigmon, S. C., Thomas, C. S., Heil, S. H., \& Higgins, S. T. (2008). voucher-based contingent reinforcement of smoking abstinence among methadone-maintained patients: A pilot study. Journal of Applied Behavior, Analysis, 41(4), 527-538. https://doi.org/ 10.1901/jaba.2008.41-527

[3] Stoops, W. W., Dallery, J., Fields, N. M., Nuzzo, P. A., Schoenberg, N. E., Martin, C. A., Casey, B., \& Wong, C. J. (2009). An internet-based abstinence reinforcement smoking cessation intervention in rural, smokers. Drug and Alcohol Dependence, 105(1), 56-62. https://doi.org/ 10.1016/ j.drugalcdep.2009.06.010

[4] Dallery, J., Stinson, L., Bolívar, H., Modave, F., Salloum, R. G., Viramontes, T. M., \& Rohilla, P. (2021). mMotiv8: A smartphone-based contingency management intervention to promote smoking cessation. Journal of Applied Behavior Analysis, 54(1), 38 53. https://doi.org/10.1002/jaba.800

[5] Weinstock, J., Capizzi, J., Weber, S. M., Pescatello, L. S., \& Petry, N. M. (2014). Exercise as an intervention for sedentary hazardous drinking college students: A pilot study. Mental Health and Physical Activity, 7(1), 5562. https://doi.org/10.1016/j.mhpa.2014.02.002

[6] Barnett, N. P., Tidey, J., Murphy, J. G., Swift, R., \& Colby, S. M. (2011). Contingency management for alcohol use reduction: A pilot study using a transdermal alcohol sensor. Drug and Alcohol Dependence, 118(2),
399. https://doi.org/10.1016/j.drugalcdep.2011.04.0 23

[7] McDonell, M. G., Leickly, E., McPherson, S., Skalisky, J., Srebnik, D., Angelo, F., Vilardaga, R., Nepom, J. R., Roll, J. M., \& Ries, R. K. (2017). A randomized controlled trial of ethyl glucuronidebased contingency management for outpatients with co-occurring alcohol use disorders and serious mental illness. The American Journal of Psychiatry, 174(4), 370377. https://doi.org/10.1176/appi.ajp.2016.1605062 7

[8] Litt, M. D., Kadden, R. M., Kabela-Cormier, E., \& Petry, N. M. (2008). Coping skills training and contingency management treatments for marijuana dependence: Exploring mechanisms of behavior change. Addiction (Abingdon, England), 103(4), 638-648. https://doi.org/10.1111/j.13600443.2008.02137.x

[9] Olmstead, T. A., \& Petry, N. M. (2009). The costeffectiveness of prize-based and voucher-based contingency management in a population of cocaine- or opioid-dependent outpatients. Drug and Alcohol Dependence, 102(1), 108115. https://doi.org/10.1016/j.drugalcdep.2009.02.0 05

[10] Metrebian, N., Weaver, T., Pilling, S., Hellier, J., Byford, S., Shearer, J., Mitcheson, L., Astbury, M., Bijral, P., Bogdan, N., Bowden-Jones, O., Day, E., Dunn, J., Finch, E., Forshall, S., Glasper, A., Morse, G., Akhtar, S., Bajaria, J., . . . Strang, J. (2018). Positive reinforcement targeting abstinence in substance misuse (PRAISe): Study protocol for a cluster RCT \& process evaluation of contingency management. Contemporary Clinical Trials, 71, 124-132. https://doi.org/10.1016/j.cct.2018.06.008 